\title{
Efectos de un programa de intervención en niños de 5 a 6 años: Evaluación del cambio proactivo en factores conductuales y cognitivos del desarrollo
}

\section{Effects of an intervention program in children aged 5-6 years: Assessment of proactive change in behavioural and cognitive development factors}

\author{
Maite Garaigordobil* y Laura Berrueco \\ Universidad del País Vasco, España
}

(Recepción: Septiembre 2007 - Aceptación: Noviembre 2007)

\begin{abstract}
Resumen
El objetivo del estudio fue evaluar los efectos de un programa de juego cooperativo en factores conductuales y cognitivos del desarrollo infantil. Se utilizó un diseño experimental pretest-intervención-postest con grupos de control. La muestra fue de 86 participantes, de 5 a 6 años, 53 experimentales y 33 de control. En la fase pretest y postest se utilizaron 5 instrumentos de evaluación. El programa consistió en una sesión de juego semanal de 75 minutos de duración durante un curso escolar. Los resultados de los análisis de varianza sugieren que el programa incrementó significativamente la capacidad cognitiva de resolución prosocial de problemas interpersonales, la conducta altruista con iguales, la fluidez verbal y la normatividad. Se confirmó un cambio significativamente superior en los experimentales que antes de la intervención tenían un nivel bajo de desarrollo. El sexo no influyó en los efectos del programa. Los resultados evidencian la relevancia del juego cooperativo en el desarrollo infantil y apoyan la idea de introducir el juego en los proyectos de la Educación Preescolar.

Palabras Claves: evaluación de programas, juegos cooperativos, desarrollo infantil.
\end{abstract}

\begin{abstract}
The purpose of this study was to evaluate the effects of a cooperative game program on the behavioral and cognitive factors of children's development. The study used an experimental pretest-interventionposttest design with control groups. The sample included 86 participants aged 5 to 6 years, 53 experimental and 33 controls. Before and after administering the program, five assessment instruments were applied. The intervention consisted of a weekly 75-minute play session throughout the school year. Results of the analysis of variance suggest that the program significantly increased the cognitive skill of prosocial interpersonal problem-solving, altruistic behavior towards peers, verbal fluency and normative behavior. The highest significant levels of improvement were found in the experimental participants who had lower levels of development at pretest, but no differences were found according to sex. The results suggest the relevance of cooperative games for children's development and support the idea of introducing such play in Preschool Education.
\end{abstract}

Key words: programs evaluation, cooperative games, children development.

El estudio tiene como objetivo evaluar el efecto de un programa de intervención configurado con juegos amistosos, de ayuda y cooperación, dirigido a niños y niñas de 5-6 años, en factores del desarrollo infantil tales como la conducta altruista (con iguales y adultos), las estrategias cognitivas

\footnotetext{
* Correspondencia a: Maite Garaigordobil, Dpto. de Personalidad, Evaluación y Tratamiento Psicológico, Facultad de Psicología, Universidad del País Vasco. Dirección: Avda. de Tolosa 70. 20018 Donostia-San Sebastián, España. Teléfonos: 943 - 015634 / Fax: 943 - 0156 70. E-mail: maite.garaigordobil@ehu.es
} 
de resolución prosocial de problemas interpersonales, la inteligencia (verbal y no verbal), la madurez neuropsicológica (atención, memoria, lenguaje comprensivo, fluidez verbal y visopercepción) y otros factores del desarrollo infantil (conceptuación, coordinación sensomotriz, contacto-comunicación, desarrollo somático, desarrollo sensorial, normatividad, reacción afectiva y reacción motriz).

Este programa dirigido a niños de edad preescolar, denominado "Programa JUEGO 4-6 años", forma parte de una línea de intervención psicológica para fomentar el desarrollo infantil, que está configurada con 4 programas de juego cooperativo, los programas JUEGO 4-6, 6-8, 8-10, y 10-12 años (Garaigordobil, 2003ab, 2004a, 2005a, 2007, Garaigordobil \& Fagoaga, 2006). Para validar los 3 programas que anteceden al programa para preescolares, se llevaron a cabo sesiones de intervención semanal durante un curso escolar. Antes y después de aplicar los programas (septiembre-junio) se aplicó una batería de instrumentos de evaluación a fin de comparar el cambio entre los grupos experimentales y control. La evaluación de los efectos de estos programas ha confirmado el impacto sistemáticamente positivo de estas experiencias lúdicas cooperativas en distintas dimensiones del desarrollo infantil.

La investigación sobre el programa para niñas y niños de 6 a 8 años constató que la intervención había potenciado: (1) un incremento de conductas sociales positivas (liderazgo, jovialidad, sensibilidad social, respeto-autocontrol); (2) una disminución de conductas sociales negativas (agresividad, apatía-retraimiento, ansiedad-timidez); (3) un aumento de la capacidad de cooperación; (4) una mejora de la comunicación intragrupo; (5) un menor empleo de estrategias cognitivas agresivas como técnica de resolución de conflictos sociales; (6) una elevación del autoconcepto; (7) la estabilidad emocional; (8) un aumento del reconocimiento del esquema corporal; y (9) una mejora en aptitudes de madurez para el aprendizaje escolar (Garaigordobil, 1992/1994, 2005a, Garaigordobil \& Echebarría, 1995, Garaigordobil, Maganto, \& Etxeberría, 1996). La investigación del programa para grupos de 8 a 10 años puso de relieve que el programa estimuló: (1) un aumento de la conducta prosocial; (2) una disminución de las conductas sociales pasivas y agresivas; (3) una mejora de la comunicación intragrupo; (4) una elevación del autoconcepto; y (5) un aumento de la creatividad (Garaigordobil, 1996, 1999, 2003b). La validación del programa para niños y niñas de 10 a 12 años confirmó que el programa había fomentado: (1) un incremento de las conductas sociales de respeto por las normas sociales, de liderazgo, asertivas y prosociales; (2) una mejora de la imagen de los compañeros del grupo; (3) un incremento de las estrategias cognitivas de interacción social asertivas; (4) una disminución de las conductas agresivas y antisociales; (5) un aumento de la estabilidad emocional; (6) una mejora del autoconcepto; (7) un incremento de la inteligencia verbal; y (8) de la creatividad (Garaigordobil, 2004ab, 2005bc).

Además de los estudios realizados con los programas JUEGO, antecedentes del programa dirigido a preescolares, otras investigaciones también han confirmado que el juego tiene efectos positivos en el desarrollo intelectual (Bjorklund \& Brown, 1998), en concreto estimula la adquisición y desarrollo del lenguaje (Brown, 1990; Clawson, 2002; Gardner, 1993; Howes, Droege, \& Matheson, 1994; Iitaka, Miyashita, \& Kakuyama, 1987; Lederer, 2002), el aprendizaje y la madurez intelectual (McCune \& Zanes, 2001; Sarle, 2000). Complementariamente, otros trabajos han constatado que, desde el punto de vista del desarrollo social, el juego estimula la capacidad de resolución cooperativa de los problemas de relación (Shores, Huster, \& Strain, 1976), la competencia social relacionada con la empatía, la toma de perspectiva y las estrategias de interacción social (Creasey, Jarvis, \& Berk, 1998; Haight \& Miller, 1993), las habilidades sociales de interacción y comunicación social (Ballou, 2001; Rosberg, 1994; Riojas-Cortez, 2000), las habilidades de negociación (Doyle, Doehring, Tessier, \& de Lorimier, 1992), y la conducta social positiva (Fall, Navelski \& Welch, 2002; Honig, 1999; Seider, 2002). Así mismo, estudios que han analizado los efectos del juego cooperativo también han ratificado que este tipo de juegos incrementan las conductas de ayuda, cooperación y de cohesión grupal (Carlson, 1999), la capacidad para compartir (Doctoroff, 1997; Rolfe, 1991), las habilidades de adaptación, interacción social y comunicación (Beilinson, 2003), las estrategias de negociación y las experiencias de compartir (Zan \& Hildebrandt, 2003), y también disminuyen el rechazo entre iguales (Mikami, Boucher, \& Humphreys, 2005). 
Tomando como referencia estos estudios, la investigación actual postula que el programa de intervención basado en el juego amistoso, de ayuda y cooperación, estimulará el desarrollo infantil en edades tempranas (5-6 años), proponiendo 7 hipótesis; en concreto se hipotetiza que el programa aumentará significativamente: (1) las estrategias cognitivas de resolución prosocial de problemas interpersonales; (2) las conductas altruistas de compartir con iguales y con adultos; (3) la inteligencia verbal y no-verbal; (4) diversos factores relacionados con la madurez neuropsicológica: lenguaje comprensivo, visopercepción, memoria icónica, fluidez verbal, y atención; así como (5) diversos factores de desarrollo: reacción afectiva, desarrollo somático, desarrollo sensorial, reacción motriz, coordinación sensomotriz, contacto-comunicación, conceptualización y normatividad. Además, se propone que: (6) el programa producirá una mejora superior en los experimentales que tengan bajos niveles de desarrollo; y (7) el género no afectará a los efectos del programa.

\section{Método}

\section{Participantes}

La muestra está constituida por 86 participantes de 5 a 6 años, distribuidos en 5 grupos pertenecientes a centros escolares públicos del norte de España. Del conjunto de la muestra 53 participantes fueron asignados aleatoriamente a la condición experimental ( 3 grupos) mientras que 33 desempeñaron la condición de control ( 2 grupos). Respecto al sexo, 47 son niños $(54,7 \%)$, mientras que 39 son niñas $(45,3 \%)$. Inicialmente los participantes seleccionados fueron 96 pero hubo una mortandad muestral de 10 sujetos, 6 de los cuales pertenecían al grupo experimental y 4 al grupo control. Los motivos por los que abandonaron el estudio fueron diversos: (a) 3 alumnos a lo largo del curso dejaron el centro escolar, no pudiendo ser evaluados en la fase postest; (b) 2 alumnos pertenecían a minorías étnicas y tenían un desfase curricular de más de un curso; y (c) 5 alumnos habían sido diagnosticados por el "Equipo de Orientación Educativa y Psicopedagógica (EOEP)" como "alumnos con necesidades educativas especiales (ACNEES)" con más de dos cursos de desfase curricular. La inclusión de los 2 alumnos de minorías y de los 5 ACNEES no fue posible debido a dificultades asociadas a la evaluación tales como: falta de concentración, fatiga, y escasa comprensión de las tareas implicadas en los tests. No obstante, los experimentales con estas características participaron en el programa de juego. Experimentales y control fueron equivalentes en términos de edad, sexo, aptitudes académicas y rendimiento. Después de seleccionar al azar los centros escolares, se realizó una reunión con los directores de los centros y los profesores de los correspondientes grupos educativos, los cuales decidieron participar en el estudio. La decisión fue realizada con la aceptación de los padres, con los que se realizó una reunión en la que se les informó de la investigación y dieron el consentimiento informado.

\section{Diseño y procedimiento}

El estudio empleó una metodología experimental, en concreto un diseño de medidas repetidas pretest-intervención-postest con grupo de control. En la fase pretest, durante las primeras semanas del curso escolar (septiembre), se administraron cinco instrumentos de evaluación para medir las variables dependientes sobre las que se hipotetizó que el programa iba a tener efectos positivos. Posteriormente, los participantes experimentales realizaron el programa de intervención que consistió en una sesión de juego semanal de 75 minutos de duración durante todo el curso escolar. Los participantes de control realizaron las actividades curriculares del programa del centro escolar, con lo que se evitó el efecto Hawthorne, ya que recibieron otro tipo de instrucción y el mismo nivel de atención. En la fase postest, durante las últimas semanas del curso escolar (junio), se administraron los mismos instrumentos de evaluación que en la fase pretest. La corrección de los tests fue ciega, sin conocimiento de la condición o las hipótesis. Para la realización de este estudio se constituyó un equipo investigador compuesto por 10 profesionales de la psicología y la educación, 3 profesoras que implementaron el programa en las aulas experimentales y 7 evaluadores ( 5 psicólogos y 2 pedagogos) que llevaron a cabo la evaluación pretest-postest. 


\section{Instrumentos}

Para medir las variables dependientes sobre las que se hipotetizó que el programa iba a tener un efecto, se elaboró una batería compuesta por 5 instrumentos de evaluación con garantías psicométricas que fue administrada antes y después de realizar el programa de juego (ver Cuadro $1)$.

Cuadro 1. Variables dependientes e instrumentos de evaluación pretest-postest

\begin{tabular}{|c|c|c|c|c|}
\hline Instrumentos & Variables dependientes & Fuente & Aplicación & Tiempo \\
\hline $\begin{array}{c}\text { TREPI } \\
\text { Test de resolución de problemas } \\
\text { interpersonales (Garaigordobil \& } \\
\text { Berrueco, 2007) }\end{array}$ & $\begin{array}{l}\text { Capacidad cognitiva de resolución } \\
\text { prosocial de problemas interpersonales }\end{array}$ & Niños/as & Individual & 15 \\
\hline $\begin{array}{c}\text { EA } \\
\text { Evaluación del altruismo } \\
\text { (Leighton, 1992) }\end{array}$ & $\begin{array}{l}\text { Conducta altruista: } \\
\text { Compartir con iguales } \\
\text { Compartir con adultos }\end{array}$ & Niños/as & $\begin{array}{l}\text { Parejas de niños/as } \\
\text { Niño/a-Evaluador }\end{array}$ & $10^{\prime}$ \\
\hline $\begin{array}{c}\text { K-BIT } \\
\text { Test breve de inteligencia } \\
\text { (Kaufman \& Kaufman, } \\
\text { 1994/1997) }\end{array}$ & $\begin{array}{l}\text { Inteligencia verbal } \\
\text { Inteligencia no verbal } \\
\text { Inteligencia total }\end{array}$ & Niños/as & Individual & $20{ }^{\prime}$ \\
\hline $\begin{array}{l}\text { CUMANIN } \\
\text { Cuestionario de madurez } \\
\text { neuropsicológica infantil } \\
\text { (Portellano, et al. 2000) }\end{array}$ & $\begin{array}{ll} & \text { Madurez neuropsicológica: } \\
\text { - } & \text { Lenguaje Comprensivo } \\
\text { - } & \text { Visopercepción } \\
\text { - } & \text { Memoria icónica } \\
\text { - } & \text { Fluidez Verbal } \\
\text { - } & \text { Atención }\end{array}$ & Niños/as & Individual & $300^{\prime}$ \\
\hline $\begin{array}{c}\text { EOD } \\
\text { Escala observacional del } \\
\text { desarrollo (Secadas, 1988/1992) }\end{array}$ & \begin{tabular}{ll} 
& \multicolumn{1}{c}{ Variables del desarrollo: } \\
- & Reacción afectiva \\
- & Desarrollo somático \\
- & Desarrollo sensorial \\
- & Reacción motriz \\
- & Coordinación sensomotriz \\
- & Contacto y comunicación \\
- & Conceptuación \\
- & Normatividad
\end{tabular} & Padres & Individual & 30 \\
\hline
\end{tabular}

TREPI. Test de resolución de problemas interpersonales (Garaigordobil \& Berrueco, 2007). La prueba permite una evaluación de las habilidades cognitivas de solución de problemas interpersonales. La tarea consiste en presentar al sujeto, a través de un dibujo y una pequeña historia, tres situaciones problemáticas que pueden surgir en la interacción social y que son cercanas a la vida del niño. Por ejemplo, la primera situación se refiere a una niña que no quiere dejar a su hermano pequeño su muñeca pero a la vez tiene miedo de que su madre le riña por ello. El sujeto evaluado tendrá que responder qué puede hacer el niño o niña de la historia en esa situación, y se otorgará un punto por 
cada respuesta prosocial de resolución de la situación problema que aporte.

EA. Evaluación del altruismo: El juego del compartimiento (Leighton, 1992ab). Evalúa el altruismo con adultos y con iguales. La tarea de altruismo con adultos consiste en realizar un juego en el cual tanto el niño como el adulto tienen que completar con fichas 25 casillas de un tablero (colocar un botón en cada casilla). El niño posee un exceso de fichas (30) mientras que el adulto tiene un déficit (20) y no puede finalizar la tarea. El adulto completa su tablero a una velocidad moderada, permitiendo que el niño termine unos tres segundos antes que él. De este modo el niño tiene la oportunidad de ver cómo el adulto coge los últimos botones de su bolsa y los coloca sobre su tablero quedándole una fila sin rellenar. En el momento en que el adulto coloca su último botón se empieza a controlar el tiempo. Las posibles reacciones del niño y las puntuaciones obtenidas pueden ser las siguientes: (a) que el niño ofrezca sus botones extras al adulto durante los primeros 75 segundos, en este caso recibe 3 puntos; (b) si tras estos 75 segundos el niño no tiene conductas de donación, el adulto dirá: "No puedo terminar mi tablero" y esperará 15 segundos más, si el niño ofrece sus botones en este espacio de tiempo recibe 2 puntos; (c) si sigue sin dar respuestas el adulto repetirá que no puede terminar de llenar sus casillas y dará otros 15 segundos, si el niño ofrece sus botones en este intervalo de tiempo recibe 1 punto; y (d) si en el tiempo máximo de 105 segundos no comparte sus botones con el adulto recibe 0 puntos. La tarea de altruismo con iguales es un test de generosidad adaptación de la tarea de Leighton. Se coloca a los niños en parejas elegidas al azar. Un miembro de la pareja sale del aula y al otro se le entregan 7 bolsitas de gominolas y dos bolsas (una con su nombre y otra con el de su pareja). Se le explica que todas las bolsitas son para él o ella, pero que si quiere, puede dar alguna o algunas a su pareja para que también tenga gominolas. Se le deja un tiempo para pensar y posteriormente el niño pone en su bolsa las gominolas que quiere para él y en la otra bolsa las que quiere dar a su pareja. En la corrección se otorgan puntos en función del número de bolsas que comparta: (a) 0 puntos si no comparte ninguna; (b) 1 punto si comparte una; (c) 2 puntos si comparte dos; (d) 3 puntos si comparte tres; (e) 5 puntos si comparte cuatro; (f) 7 puntos si comparte cinco; (g) 9 puntos si comparte seis; y (h) 11 puntos si comparte las siete.

K-BIT. Test breve de inteligencia (Kaufman \& Kaufman, 1994/1997). La prueba evalúa la inteligencia verbal, no verbal y global. Consta de 2 subtest: vocabulario y matrices. Vocabulario expresivo mide habilidades verbales relacionadas con el aprendizaje escolar (pensamiento cristalizado) apoyándose en el conocimiento de palabras. La tarea consiste en nombrar un objeto representado gráficamente. Matrices mide habilidades no verbales y capacidad para resolver nuevos problemas (pensamiento fluido), a partir de la aptitud del sujeto para percibir relaciones y completar analogías visuales. La tarea consiste en comprender la relación existente entre varios estímulos representados gráficamente. En los ítems más sencillos el sujeto tiene que elegir entre cinco figuras propuestas la que mayor relación posee con la que se propone como estímulo (un coche con un camión, un perro con un hueso). En otro conjunto de ítems, el sujeto debe elegir entre seis u ocho figuras la que mejor completa una analogía visual (sombrero es a cabeza como zapato es a pie).

CUMANIN. Cuestionario de madurez neuropsicológica infantil (Portellano et al. 2000). El cuestionario permite valorar la madurez neuropsicológica de niños de 3 a 6 años mediante 13 escalas, de las cuales para esta investigación se seleccionaron 5: atención, fluidez verbal, lenguaje comprensivo, memoria icónica y visopercepción. Para la prueba de atención se entrega al sujeto una ficha con figuras geométricas. El ejercicio consiste en tachar todas las figuras iguales al modelo (un cuadrado) durante 30 segundos. En la prueba de fluidez verbal, el niño deberá crear una frase, cuanto más larga mejor, a partir de una palabra o dos palabras dadas (coche, televisión-tigre). En lenguaje comprensivo el niño escucha una historia y a continuación se le plantean 9 preguntas sobre el contenido de esa historia. Para la prueba de memoria se presenta al sujeto una lámina con 10 dibujos durante un minuto. Posteriormente se le pide que nombre todos los dibujos que recuerde. En la escala de visopercepción el sujeto tiene que reproducir 15 figuras de dificultad creciente.

EOD. Escala observacional del desarrollo (Secadas, 1988/1992). La escala evalúa el estado del desarrollo en que se encuentra un sujeto en un período determinado. La EOD tiene un total de 69 enunciados que conforman ocho escalas: (1) reacción afectiva, (se vuelve vergonzoso y muestra 
pudor); (2) desarrollo somático (salta a la cuerda y a la goma); (3) desarrollo sensorial (puede graduar los colores de más claro a más oscuro); (4) reacción motriz (se da impulso a sí mismo al columpiarse); (5) coordinación sensomotriz (usa las tijeras espontáneamente cuando quiere cortar papel); (6) contacto y comunicación (prefiere reunirse con otros compañeros para jugar); (7) conceptuación (conoce el significado de mañana, tarde, noche, verano, invierno); y (8) normatividad (entiende y respeta reglas sencillas del juego). La escala es cumplimentada por los padres que leen los enunciados y responden si describen o no el comportamiento de sus hijos e hijas.

\section{Intervención o Tratamiento}

La intervención consistió en la realización de una sesión de juego cooperativo semanal de 75 minutos de duración durante todo el curso escolar. Estas sesiones fueron dirigidas por el profesor o profesora habitual del grupo y se realizaron en el mismo horario semanal y en el mismo espacio físico, un aula de psicomotricidad o gimnasio. Las actividades del programa de intervención tienen como principales objetivos estimular: (1) procesos de comunicación dentro del grupo (exponer, escuchar, dialogar, negociar, tomar decisiones por consenso...); (2) las interacciones prosociales (ayudar, cooperar, compartir, consolar...); y (3) la expresión de emociones a través de la palabra, el dibujo, la pintura, la dramatización... Los juegos que contiene el programa estimulan la comunicación, la cohesión, la confianza, teniendo en su base la idea de aceptarse, cooperar y compartir, jugando e inventando juntos. Los juegos incluidos en este programa tienen 5 características estructurales: (1) la participación, ya que en estos juegos todos los miembros del grupo participan, nunca hay eliminados, ni nadie pierde; el objetivo consiste en alcanzar metas grupales, y para ello cada participante tiene un papel necesario para la realización del juego; (2) la comunicación, porque todos los juegos del programa estructuran procesos de comunicación dentro del grupo que implican escuchar, dialogar, tomar decisiones, negociar...; (3) la cooperación, ya que los juegos del programa estimulan que los jugadores se den ayuda mutuamente para contribuir a un fin común, a una meta de grupo; (4) la ficción y creación, porque se juega a representar, a hacer el "como si" de la realidad, como si fuéramos leones, magos..., así como a combinar estímulos para crear algo nuevo; y (5) la diversión, ya que con estos juegos se intenta que los miembros del grupo se diviertan interactuando de forma positiva, constructiva y creativa con sus compañeros.

La dinámica estructural de una sesión de juego se configura con tres fases: apertura, desarrollo de la secuencia de juego, y cierre. Las sesiones comienzan con la fase de apertura en la que los miembros del grupo están sentados en el suelo y en posición circular. Con esta organización grupal se comentan brevemente los objetivos de los juegos cooperativos: divertirse, hacer amigos, aprender a ayudarse, a colaborar con los compañeros para hacer cosas en equipo, a escucharse, a ser respetuosos con las ideas de otros... Habitualmente, el adulto comunica al grupo la apertura de la sesión de juego cooperativo, y en las primeras sesiones llama la atención sobre los objetivos generales de la experiencia, pero después de dos o tres sesiones preguntará al grupo por los objetivos con la finalidad de fomentar la comunicación circular (feedback-retroalimentación) sobre los objetivos del programa de juego. En la segunda fase, la fase de desarrollo de la secuencia de juegos, se realizan sucesivamente 2 ó 3 actividades lúdicas cooperativas. Los miembros del grupo están sentados en el suelo en posición circular y el adulto expone las instrucciones del primer juego. Cuando los miembros del grupo han comprendido perfectamente en qué consiste el juego, qué deben hacer y con qué procedimiento, lo llevan a cabo. Posteriormente, vuelven al círculo y en esta posición de relajación el adulto da las instrucciones para desarrollar el segundo juego, y así sucesivamente con los juegos que configuren la sesión. Después de realizar la secuencia de juego, se inicia la fase de cierre en la que se lleva a cabo una reflexión y diálogo sobre lo sucedido en la sesión de juego: sentimientos tenidos en la experiencia, participación, rechazos, respeto por las reglas, cooperación... Para la realización del cierre los jugadores se sientan en el suelo en posición circular. El adulto que dirige la intervención formula preguntas a los miembros del grupo (cómo se han sentido en la sesión de juego, si han cooperado, si se han divertido, si han tenido problemas...). En esta fase de diálogo se analiza todo lo relacionado con la acción y las interacciones acontecidas en los juegos realizados. 
El cierre es un ejercicio de reflexión en el que los miembros del grupo verbalizan tanto los aspectos positivos de la experiencia, como los problemas surgidos. En esta fase el adulto además de promover la comunicación dentro del grupo respecto a la experiencia vivida, aporta refuerzo social, valoración verbal en relación a las conductas de ayuda, diálogo o cooperación observadas, enfatizando la valía de los productos elaborados....

Con la finalidad de clarificar las características del programa, de los 140 juegos que configuran el programa para niños de 4 a 6 años (Garaigordobil, 2007), a modo de ejemplo se presentan las fichas técnicas de dos juegos (ver Fichas Técnicas de los Juegos 27 y 40).

Ficha técnica del Juego 27. Un camino con obstáculos.

\begin{tabular}{|c|c|}
\hline \multicolumn{2}{|r|}{ 27. UN CAMINO CON OBSTÁCULOS } \\
\hline \multicolumn{2}{|r|}{ Objetivos: Comunicación. Ayuda. Empatía. } \\
\hline Desarrollo Afectivo & $\begin{array}{l}\text { - Placer de ayudar y ser ayudado, placer de llegar a la meta. } \\
\text { - Control emocional: afrontamiento y superación de sentimientos de inseguridad y temor derivados de } \\
\text { desplazarse a ciegas. } \\
\text { - Empatía hacia los estados emocionales de otro ser humano: sensibilidad hacia las personas invidentes. } \\
\text { - Sentimientos de aceptación: cada jugador tiene un papel necesario para la realización del juego. }\end{array}$ \\
\hline $\begin{array}{c}\text { Desarrollo } \\
\text { Social }\end{array}$ & $\begin{array}{l}\text { - Comunicación verbal: expresión y hábitos de escucha activa. } \\
\text { - Conductas de ayuda: para facilitar el desplazamiento del invidente. }\end{array}$ \\
\hline $\begin{array}{l}\text { Desarrollo } \\
\text { Intelectual }\end{array}$ & $\begin{array}{l}\text { - Simbolización. } \\
\text { - Atención. }\end{array}$ \\
\hline Desarrollo Psicomotriz & $\begin{array}{l}\text { - Funciones psicomotrices: orientación espacial, noción de dirección sin referencia visual, esquema } \\
\text { corporal, percepción auditiva. }\end{array}$ \\
\hline
\end{tabular}

\section{Descripción de la actividad}

Los jugadores se agrupan por parejas, uno representa a un ciego y el otro a un guía. El aula se dispone llena de objetos que representan obstáculos. El guía debe ayudar a su compañero ciego a desplazarse por el aula mediante instrucciones verbales de orientación espacial (adelante, a la derecha, a la izquierda, atrás...) hasta llegar a una meta señalada previamente. Cuando la pareja llega a la meta, cambian los roles y se desplazan hasta la línea de salida.

Notas: Se sugiere realizar el juego al final del último curso de educación infantil. Si se realiza el juego en un nivel de edad anterior, el guía da la mano al ciego para ayudar en su desplazamiento.

\section{Debate}

¿Cómo os habéis sentido en este juego? ¿Ha sido divertido? ¿Habéis tenido miedo a desplazaros a ciegas por el aula en la que había muchos obstáculos con los que podíais chocar? ¿Qué habéis sentido al ayudar a vuestro compañero? ¿Y al ser ayudado por él? ¿Os habéis chocado mucho? ¿Cómo os habéis sentido cuando esto ha sucedido? ¿Cómo pensáis que se sienten las personas que no pueden ver?

\begin{tabular}{c|c|c} 
Materiales & Tiempo & Estructura grupal \\
1 venda para los ojos por jugador. & 30 minutos & PR \\
\hline
\end{tabular}


Ficha técnica del Juego 40. Puzzles con nuestros retratos.

\begin{tabular}{|c|c|c|c|}
\hline \multicolumn{4}{|c|}{ 40. PUZZLES CON NUESTROS RETRATOS } \\
\hline \multicolumn{4}{|c|}{ Objetivos: Comunicación. Cohesión grupal. Cooperación. } \\
\hline Desarrollo Afectivo & \multicolumn{3}{|c|}{$\begin{array}{l}\text { - Placer de conseguir la meta, placer de ayudar y ser ayudado. } \\
\text { - Sentimientos de aceptación: cada jugador tiene un papel necesario para la realización del juego. } \\
\text { - Autoafirmación del Yo. }\end{array}$} \\
\hline $\begin{array}{l}\text { Desarrollo } \\
\text { Social }\end{array}$ & \multicolumn{3}{|c|}{$\begin{array}{l}\text { - Comunicación verbal: hábitos de escucha activa. } \\
\text { - Cohesión grupal: sentimientos de pertenencia. }\end{array}$} \\
\hline $\begin{array}{l}\text { Desarrollo } \\
\text { Intelectual }\end{array}$ & \multicolumn{3}{|c|}{$\begin{array}{l}\text { - Capacidad de síntesis: configuración de un todo mediante la integración de partes. } \\
\text { - Atención. }\end{array}$} \\
\hline Desarrollo Psicomotriz & \multicolumn{3}{|c|}{$\begin{array}{l}\text { - Funciones psicomotrices: coordinación visomotora, percepción visual, anticipación a la relación partes- } \\
\text { todo, orientación espacial, lateralidad. }\end{array}$} \\
\hline \multicolumn{4}{|c|}{ Descripción de la actividad } \\
\hline \multicolumn{4}{|c|}{$\begin{array}{l}\text { En este juego se solicita que cada equipo reconstruya cooperativamente cuatro fotografías de } \\
\text { los miembros del grupo. Después de formar equipos de } 4 \text { jugadores, se entrega a cada jugador } \\
\text { un sobre que contiene } 3 \text { piezas de distintas fotografías y se da la siguiente consigna de juego: } \\
\text { "Jugando en cada equipo debéis construir unos puzzles de fotografías de niños y niñas de esta } \\
\text { clase. Voy a daros a cada uno un sobre que contiene } 3 \text { piezas de las fotos. Distribuidas entre los } \\
\text { cuatro sobres están las piezas que pueden formar } 4 \text { fotografías de niños o niñas de esta clase, } \\
\text { fotografías como ésta (se muestra una fotografía modelo). Cada uno debe formar una fotografía } \\
\text { en frente de él, pero el juego termina cuando entre los } 4 \text { consigáis construir las } 4 \text { fotografías. Pero } \\
\text { para ello debéis dar piezas a los compañeros del equipo y recibir piezas de ellos. Además tenéis } \\
\text { que seguir las siguientes reglas: Podéis dar piezas que tenéis a vuestros compañeros de equipo } \\
\text { a los que les pueda hacer falta para completar su fotografía, pero no podéis quitar piezas a los } \\
\text { otros. Podéis pedir piezas si veis que otro compañero del equipo tiene un trozo de fotografía que } \\
\text { necesitáis, pero tenéis que esperar a que os ceda la pieza voluntariamente. Es muy importante que } \\
\text { estéis atentos para ver qué piezas no necesitáis y ofrecerlas a quien le pueda ir bien para la foto que } \\
\text { está reconstruyendo". Al final todos los miembros del grupo observan los puzzles. }\end{array}$} \\
\hline \multicolumn{4}{|c|}{$\begin{array}{l}\text { Debate } \\
\text { ¿Cómo os habéis sentido en este juego? ¿Ha sido divertido? ¿Ha sido fácil compartir las piezas para reconstruir vuestros retratos? } \\
\text { ¿Qué ha ayudado para conseguir el objetivo del juego? ¿Cómo os habéis sentido al ver terminadas vuestras fotografías? ¿Ha habido } \\
\text { algún problema? ¿Cuál? ¿Cómo se ha resuelto? }\end{array}$} \\
\hline \multicolumn{2}{|c|}{$\begin{array}{l}\text { Materiales } \\
\text { Por equipo: } 4 \text { fotografías en color de } 4 \text { miembros del grupo en tamaño folio, cada } \\
\text { una de las cuales se cortará en } 3 \text { trozos y } 4 \text { sobres. }\end{array}$} & $\begin{array}{l}\text { Tiempo } \\
30 \text { minutos }\end{array}$ & $\begin{array}{l}\text { Estructura } \\
\text { grupal } \\
\text { PG }\end{array}$ \\
\hline
\end{tabular}

\section{Resultados}

Efectos del programa en variables conductuales y cognitivas del desarrollo infantil

Para analizar los efectos del programa, con los datos obtenidos en las pruebas pretest-postest se calcularon las medias y las desviaciones típicas en relación a cada variable objeto de estudio, para los participantes experimentales y control, llevándose a cabo análisis de varianza multivariados y univariados (MANOVAs, ANOVAs) con las puntuaciones pretest, postest, así como con las diferencias pretest-postest, y obteniendo las covarianzas (MANCOVAs, ANCOVAs) en aquellas variables en las que se encontraron diferencias significativas entre ambas condiciones en la fase pretest. Los resultados se presentan en la Tabla 1. 


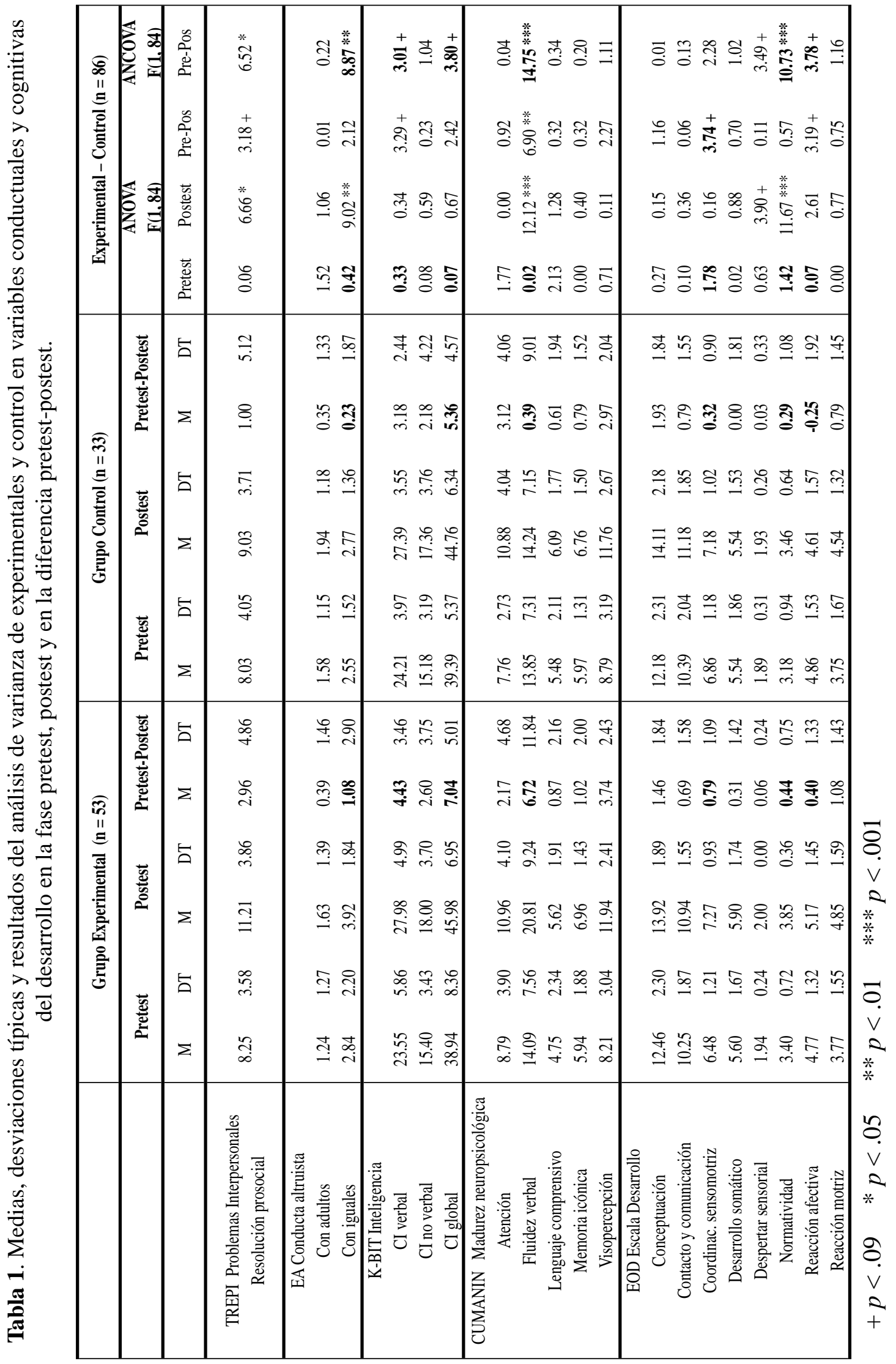


Los resultados obtenidos han puesto de relieve que este programa de juego cooperativo ha estimulado significativamente cambios positivos en varios factores conductuales y cognitivos del desarrollo infantil (ver Tabla 1). En la capacidad de resolución prosocial de problemas interpersonales, los resultados del ANCOVA pretest-postest muestran que los participantes experimentales aumentaron más $(M=2.96)$ que los de control $(M=1.00)$, siendo estas diferencias estadísticamente significativas, $F(1,84)=6.52, p<.05$.

En la conducta altruista (con iguales y con adultos), el MANOVA pretest no evidenció diferencias significativas entre experimentales y control, $F(1,84)=0.97, p>.05$, sin embargo, los resultados del MANCOVA pretest-postest, $F(1,84)=4.76, p<.05$, confirmaron diferencias significativas entre ambas condiciones. No obstante, el análisis de cada variable de forma independiente únicamente ratificó diferencias en la conducta altruista con los iguales, en la que se observó un aumento mayor en los experimentales $(M=1.08)$ que en los de control $(M=0.23)$, y el ANCOVA pretest-postest puso de relieve diferencias estadísticamente significativas entre condiciones, $F(1,84)=8.87, p<$ .01 .

En inteligencia, el MANOVA pretest no evidenció diferencias significativas entre experimentales y control, $F(1,84)=0.35, p>.05$, pero tampoco se encontraron diferencias en el MANCOVA pretest-postest, $F(1,84)=2.11, p>.05$. Los resultados del ANCOVA para cada una de las variables constataron un aumento tendencialmente significativo en la inteligencia verbal, $F(1,84)=3.01, p$ $<.09$, con incrementos pretest-postest superiores en los experimentales $(M=4.43)$ que en los de control $(M=3.18)$, así como en la inteligencia global, $F(1,84)=3.80, p<.09$, con un aumento mayor en los experimentales $(M=7.04)$ que en los de control $(M=5.36)$.

En factores relacionados con la madurez neuropsicológica, los resultados del MANOVA pretest no evidenció diferencias significativas entre experimentales y control, $F(1,84)=1.12, p>.05$, sin embargo, los resultados del MANOVA pretest-postest, $F(1,84)=2.91, p<.05$, como del MANCOVA pretest-postest, $F(1,84)=3.45, p<.01$, encontraron diferencias significativas entre ambas condiciones. No obstante, los ANCOVAs pretest-postest para cada una de las variables analizadas de forma independiente únicamente confirmaron un cambio estadísticamente significativo en la fluidez verbal, $F(1,84)=14.75, p<.001$, con puntuaciones superiores en los experimentales $(M=6.72)$ frente a los de control $(M=0.39)$.

Finalmente, en el conjunto de los factores del desarrollo evaluados por los padres, los resultados del MANOVA pretest no evidenció diferencias entre experimentales y control, $F(1,84)=0.79, p$ $>.05$, sin embargo, los resultados del MANOVA pretest-postest, $F(1,84)=2.51, p<.05$, y del MANCOVA pretest-postest, $F(1,84)=3.45, p<.01$, pusieron de relieve diferencias significativas entre ambas condiciones. Los ANCOVAs pretest-postest para cada una de las variables (ver Tabla 1) constataron un cambio estadísticamente significativo en normatividad, $F(1,84)=10.73, p<.001$, con incrementos mayores en los experimentales $(M=0.44)$ que en los de control $(M=0.29)$, y una mejora tendencialmente significativa en coordinación sensomotriz, $F(1,84)=3.74, p<.09$, con aumentos superiores en los experimentales $(M=0.74)$ que en los de control $(M=0.32)$, así como en reacción afectiva, $F(1,84)=3.78, p<.09$, en la que los experimentales muestran un aumento $(M=$ $0.40)$, mientras que los de control tienen un decremento $(M=-0.25)$.

En síntesis, los resultados sugieren que el programa incrementó significativamente: (1) la capacidad para buscar soluciones prosociales a problemas interpersonales, de interacción, empleando para ello posturas de acercamiento, estrategias de negociación, evitación de agresividad...; (2) las conductas prosociales altruistas, conductas generosas de compartir con los iguales; (3) la fluidez verbal relacionada con la producción de frases más largas a partir de una palabra estímulo; así como (4) la normatividad o conocimiento y cumplimiento de las normas sociales. Complementariamente, el programa estimuló tendencialmente una mejora de: (5) la inteligencia verbal, relacionada con el lenguaje, con el conocimiento de palabras (pensamiento cristalizado) y la inteligencia global; (6) la coordinación sensomotriz, es decir, la coordinación mediacional con instrumentos y la coordinación figuracional (representativa, constructora y gráfica); y (7) la madurez afectiva o capacidad para dar respuestas afectivas acordes con su nivel evolutivo. 


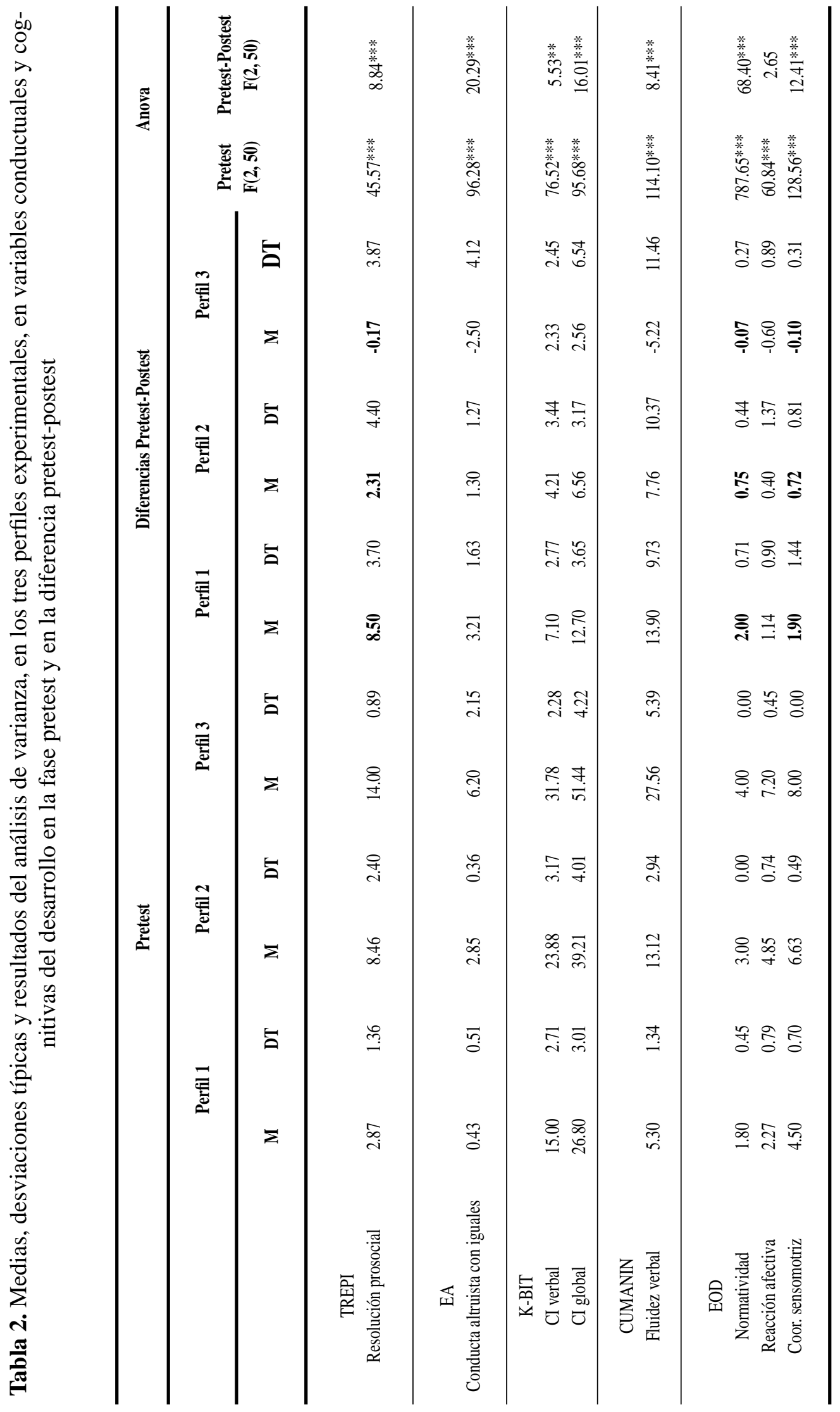




\section{Efectos del programa en los participantes experimentales con bajo nivel de desarrollo}

Para evaluar si el programa fue especialmente eficaz para los participantes experimentales que antes de iniciar la intervención tenían bajo nivel de desarrollo, en las variables en las que el programa tuvo efectos significativos, los experimentales fueron clasificados en 3 perfiles o categorías en función de sus puntuaciones pretest. El perfil 1 incluye sujetos que obtuvieron puntuaciones directas inferiores a un percentil 20; el perfil 2 engloba a los sujetos con puntuaciones correspondientes a percentiles entre 20 y 80 , y el perfil 3 incluye a los sujetos con puntuaciones superiores a un percentil 80. Con la finalidad de comprobar si el programa ejerció un efecto diferencial en los experimentales de distintos niveles de desarrollo se realizaron análisis descriptivos e inferenciales (ANOVAs) en función del perfil, en la fase pretest y en las diferencias pretest-postest (ver Tabla 2).

Como se puede observar en la Tabla 2, los resultados de los ANOVAs pretest en todas las variables confirman que había diferencias significativas entre los perfiles, es decir, partían de niveles muy distintos, lo que pone de relieve la correcta categorización de los participantes en los 3 perfiles de desarrollo: bajo, medio y alto. Los resultados de los ANOVAs de las diferencias pretestpostest constatan diferencias estadísticamente significativas entre los perfiles en todas las variables, excepto en reacción afectiva. El análisis de las diferencias pretest-postest permite confirmar que la intervención fue significativamente más eficaz para aquellos participantes que antes de la intervención tenían bajos niveles de desarrollo, es decir, baja capacidad de resolución prosocial de problemas interpersonales, bajo nivel de conducta altruista con los compañeros del grupo, bajo nivel de inteligencia, baja capacidad de fluidez verbal, así como bajo nivel de normatividad y de coordinación sensomotriz.

\section{Influencia del género en los efectos del programa}

Con la finalidad de explorar si el programa tuvo un efecto diferencial en función del sexo, se realizaron análisis de varianza en las variables del desarrollo infantil en las que el programa había sido significativamente eficaz. Se llevaron a cabo análisis descriptivos (medias y desviaciones típicas) y de varianza (ANOVAs, ANCOVAs) en la fase pretest y en las diferencias pretest-postest en función del sexo, cuyos resultados se exponen en la Tabla 3.

Como se puede observar en la Tabla 3, en la fase pretest no había diferencias entre los niños y las niñas experimentales en ninguna de las variables objeto de estudio, y tampoco se encontraron diferencias estadísticamente significativas entre ambos sexos en el cambio que experimentaron por efecto del programa. Por lo tanto, el programa no tuvo un impacto diferencial en función del género.

\section{Discusión}

Los resultados sugieren que el programa incrementó significativamente la capacidad para buscar soluciones prosociales a problemas de interacción, empleando para ello posturas de acercamiento, estrategias de negociación, evitación de agresividad..., por consiguiente se confirma la hipótesis 1. Por otro lado, también se encontró que la intervención fomentó significativamente un aumento de las conductas prosociales altruistas, conductas generosas de compartir con los iguales, ratificándose parcialmente la hipótesis 2 , ya que no se evidenció un incremento significativo de las conductas altruistas con los adultos.

En tercer lugar, los resultados han mostrado que el programa de juego cooperativo aumentó de forma tendencial la inteligencia verbal, relacionada con el lenguaje, con el conocimiento de palabras (pensamiento cristalizado) y la inteligencia global, por lo que se ratifica parcialmente la hipótesis 3. Además, los resultados sugieren que el programa tuvo un efecto significativo en algunos factores de la madurez neuropsicológica como la fluidez verbal relacionada con la producción de frases más largas a partir de una palabra estímulo, sin embargo, no afectó a otras variables como el lenguaje comprensivo, la visopercepción, la memoria icónica, y atención, por lo que se ratifica parcialmente la hipótesis 4.

Finalmente, los resultados permiten concluir que el programa aumentó significativamente la 


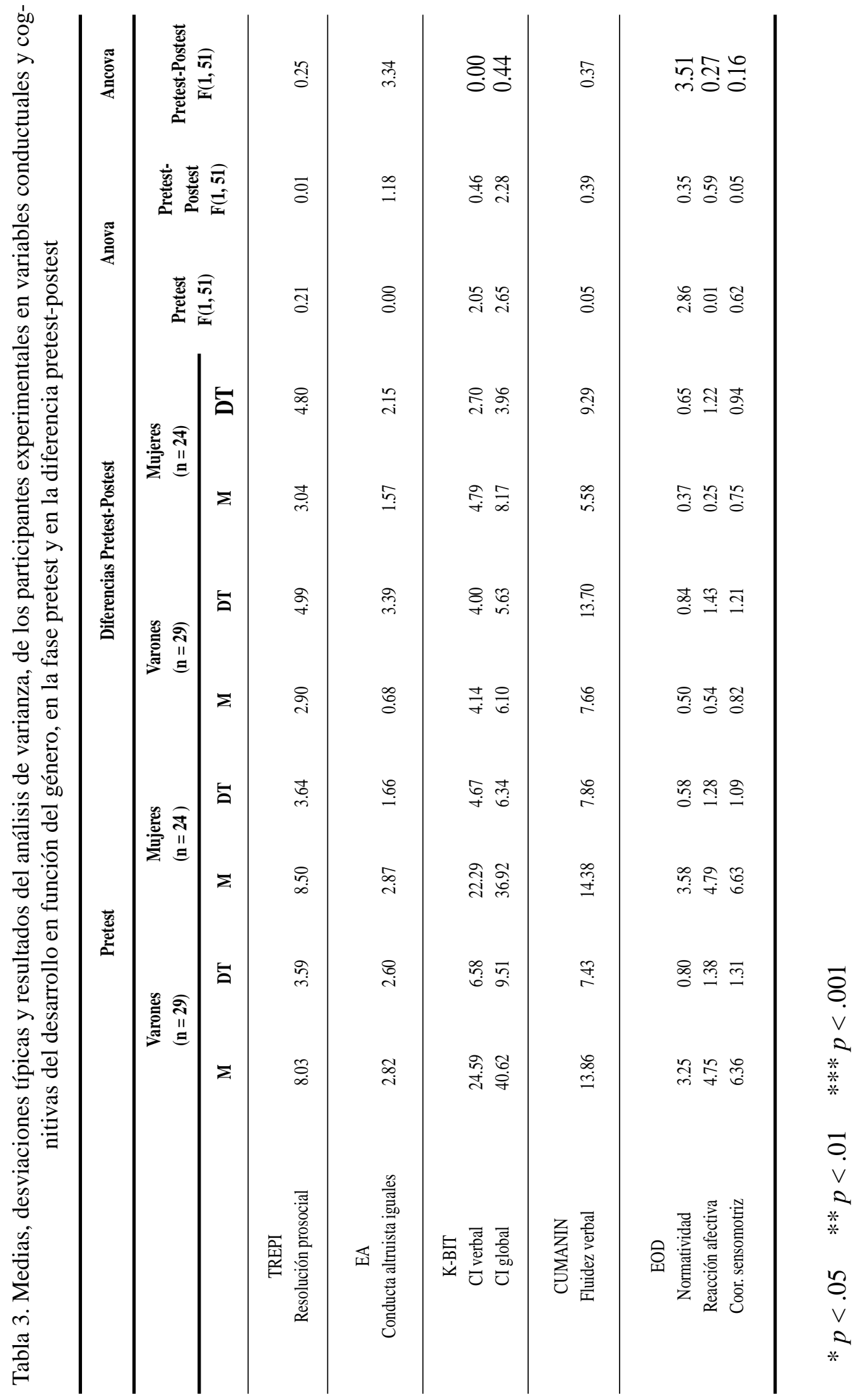


normatividad, es decir, el conocimiento y cumplimiento de las normas sociales, y aunque de forma tendencial también estimuló la coordinación sensomotriz (la coordinación mediacional con instrumentos y la coordinación figuracional representativa, constructora y gráfica), y la madurez afectiva (la reacción afectiva o capacidad para dar respuestas afectivas acordes con su nivel evolutivo), por lo que se ratifica parcialmente la hipótesis 5, ya que el programa no afectó en otros factores del desarrollo como: desarrollo somático, desarrollo sensorial, reacción motriz, contacto-comunicación y conceptualización.

Los resultados obtenidos en este estudio apuntan en la misma dirección que otros estudios que han encontrado efectos positivos del juego en factores relacionados con la socialización infantil, como son las estrategias cognitivas de interacción social positivas, la conducta social positiva con iguales, la capacidad para respetar las normas sociales (Beilinson, 2003; Carlson, 1999; Creasey et al., 1998; Doctoroff, 1997; Doyle et al., 1992; Fall et al., 2002; Honig, 1999; Seider, 2002; Haight \& Miller, 1993; Garaigordobil, 1996, Garaigordobil \& Echebarría, 1995, Garaigordobil et al., 1996, Garaigordobil \& Fagoaga, 2006; Rolfe, 1991; Shores et al., 1976; Zan \& Hildebrandt, 2003). Y así mismo ratifican los estudios que han encontrado efectos positivos del juego en la inteligencia verbal, en la adquisición y desarrollo del lenguaje (Brown, 1990; Clawson, 2002; Gardner, 1993; Garaigordobil, 2005bc; Garaigordobil \& Fagoaga, 2006; Howes et al., 1994; Iitaka et al., 1987; Lederer, 2002).

Por otro lado, los resultados permiten confirmar la hipótesis 6 que planteaba que el programa iba a producir una mejora significativamente superior en los experimentales que antes de comenzar el programa tuvieran bajos niveles de desarrollo, ya que éstos han mejorado significativamente más en prácticamente todas las variables en relación a los participantes que en la fase pretest tenían un nivel de desarrollo medio o alto. En último lugar, se confirma la hipótesis 7 ya que el género no ha afectado a los efectos del programa, habiendo mejorado los niños y niñas de forma similar en todas las variables evaluadas.

Entre los factores que pueden explicar los efectos del programa pueden sugerirse tanto las propias características estructurales de los juegos que contiene el programa (participación, comunicación, ayuda, cooperación...) con el tipo de interacciones que potencian entre los miembros del grupo, como la importancia metacognitiva de las fases de debate y diálogo que se llevan a cabo después de cada actividad. Así, se puede considerar que ambos factores, tanto la acción moral implícita en las actividades como la metacognición de la misma estructurada en los debates, son fuente de desarrollo moral y determinan en cierta medida los resultados obtenidos.

Las conclusiones del estudio realizado ponen de manifiesto que el juego es una herramienta metodológica relevante para fomentar el desarrollo infantil, especialmente el desarrollo social, confirmando que el juego temprano y variado contribuye de modo positivo a todos los aspectos del crecimiento y desarrollo humano. Igual que en los tres programas que anteceden al presente estudio, los resultados del programa diseñado para preescolares permiten confirmar la influencia positiva del juego cooperativo en el desarrollo de niños y niñas de esta edad. Los resultados obtenidos en las sucesivas evaluaciones de programas de juego cooperativo apoyan la idea de introducir el juego y la conducta prosocial como elementos integrantes de los Proyectos Educativos tanto en Educación Infantil (preescolar) como en Educación Primaria, y subrayan la necesidad de que éstos sean ejes transversales del currículum que impregnen todas las áreas curriculares.

Como limitación de este estudio cabe destacar la ausencia de evaluación de las características de los adultos que han dirigido la intervención y su influencia sobre los efectos del programa. Entre las líneas futuras de investigación se pueden plantear: (1) evaluar el efecto del programa en interacción con las características del adulto que dirige la intervención; (2) evaluar los efectos de este programa de intervención en otros factores de la personalidad infantil, por ejemplo, en el autoconcepto, en la conducta agresiva, en la creatividad..., o (3) comparar los efectos de este programa de juego cooperativo con los efectos de programas de juego competitivo. 


\section{Referencias}

Ballou, K.J. (2001). The effects of a drama intervention on communication skills and learning attitudes of at risk sixth grade students. Dissertation Abstracts International: Section A: Humanities \& Social Sciences, 61(10-A), 3828.

Beilinson, J.S. (2003). Facilitating peer group entry in kindergartners with impairment in social communication. Language, Speech, \& Hearing Services in Schools, 34(2), 154-166.

Bjorklund, D.F., \& Brown, R.D. (1998). Psysical play and cognitive development: Integrating activity, cognition and education. Child Development, 69(3), 604-606.

Brown, V. (1990). Drama as an integral part of the early childhood curriculum. Design for Arts in Education, 91(6), 26-33.

Carlson, J.M. (1999). Cooperative games: A pathway to improving health. Professional School Counseling, 2(3), 230-236.

Clawson, M. (2002). Play of language: Minority children in an early childhood setting. En J. L. Roopnarine (Ed.), Conceptual, social-cognitive, and contextual issues in the fields of play (Vol. 4, pp. 93-116). Westport, CT: Ablex.

Creasey, G.L., Jarvis, P.A., \& Berk, L.E. (1998). Play and social competence. En O.N. Saracho., \& B. Spodek (Eds.), Multiple perspectives on play in early childhood education. SUNY series, early childhood education. Inquiries and insights (pp. 116-143). New York: State University of New York Press.

Doctoroff, S. (1997). Sociodramatic script training and peer role prompting: Two tactics to promote sociodramatic play and peer interaction. Early Child Development \& Care, 136, $27-43$.

Doyle, A.B., Doehring, P., Tessier, O., \& de Lorimier, S. (1992). Transitions in children's play: A sequential analysis of states preceding and following social pretense. Developmental Psychology, 28(1), 137-144.

Fall, M., Navelski, L.F., \& Welch, K.K. (2002). Outcomes of a play intervention for children identified for special education services. International Journal of Play Therapy, 11(2), 91-106.

Garaigordobil, M. (1994). Diseño y evaluación de un programa lúdico de intervención psicoeducativa con niños de 6-7 años. Bilbao: Servicio Editorial de la Universidad del País Vasco (Microfichas de Tesis Doctorales). Tesis doctoral presentada en 1992.

Garaigordobil, M. (1996). Evaluación de una intervención psicoeducativa en sus efectos sobre la conducta prosocial y la creatividad. Madrid: Centro de Publicaciones del Ministerio de Educación y Cultura. CIDE. Colección Investigación nº 127.

Garaigordobil, M. (1999). Assessment of a cooperative-creative program of assertive behavior and selfconcept. Spanish Journal of Psychology, 2(1), 3-10.

Garaigordobil, M. (2003a). Intervención psicológica para desarrollar la personalidad infantil. Juego, conducta prosocial y creatividad. Madrid: Pirámide. (volumen 1)

Garaigordobil, M. (2003b). Programa Juego 8-10 años. Juegos cooperativos y creativos para grupos de niños de 8 a 10 años. Madrid: Pirámide. (volumen 3)

Garaigordobil, M. (2004a). Programa Juego 10-12 años. Juegos cooperativos y creativos para grupos de niños de 10 a 12 años. Madrid: Pirámide. (volumen 4)

Garaigordobil, M. (2004b). Intervención psicológica en la conducta agresiva y antisocial con niños. Psicothema, 16(3), 429-435.

Garaigordobil, M. (2005a). Programa Juego 6-8 años. Juegos cooperativos y creativos para grupos de niños de 6 a 8 años. Madrid: Pirámide. (volumen 2) 
Garaigordobil, M. (2005b). Diseño y evaluación de un programa de intervención socioemocional para promover la conducta prosocial y prevenir la violencia. Madrid: Publicaciones del Ministerio de Educación y Ciencia. Colección Investigación nº 160.

Garaigordobil, M. (2005c). Prosocial and creative play: Effects of a program on the verbal and nonverbal intelligence of children aged 10-11 years. International Journal of Psychology, 40(3), 176 $-188$.

Garaigordobil, M. (2007). Programa Juego 4-6 años. Juegos cooperativos y creativos para grupos de niños de 4 a 6 años. Madrid: Pirámide. (volumen 5)

Garaigordobil, M., \& Berrueco, L. (2007). TREPI. Test de resolución de problemas interpersonales. En M. Garaigordobil (Ed.), Programa Juego 4-6 años. Juegos cooperativos y creativos para grupos de niños de 4 a 6 años. Madrid: Pirámide.

Garaigordobil, M., \& Echebarría, A. (1995). Assessment of a peer-helping game program on children's development. Journal of Research in Childhood Education, 10(1), 63-69.

Garaigordobil, M., \& Fagoaga, J.M. (2006). Juego cooperativo para prevenir la violencia en los centros educativos: Evaluación de programas de intervención para educación infantil, primaria y secundaria. Madrid: Centro de Publicaciones del Ministerio de Educación y Ciencia.

Garaigordobil, M., Maganto, C., \& Etxeberría, J. (1996). Effects of a cooperative game program on socio-affective relationships and group cooperation capacity. European Journal of Psychological Assessment, 12(2), 140-151.

Gardner, H. (1993). Frames of mind. The theory of multiple intelligences (10th anniversary ed.). New York: Basic Books.

Haight, W.L., \& Miller, P.J. (1993). Pretending at home: Early development in a sociocultural context. Albany: State University of New York Press.

Honig, A.S. (1999). Creating a prosocial curriculum. Montessori Life, 11(2), 35-37.

Howes, C., Droege, K., \& Matheson, C. C. (1994). Play and communication processes within longand short-term friendship dyads. Journal of Social and Personal Relationships, 11, 401-410.

Iitaka, K., Miyashita, K., \& Kakuyama, T. (1987). Language intervention to a retarded child through cognitive training and pretend play. RIEEC Report, 36(2), 99-109.

Kaufman, A.S., y Kaufman, N.L. (1997). Test breve de inteligencia de Kaufman K-BIT. Madrid: TEA (trabajo original publicado en 1994).

Lederer, S. H. (2002). Collaborative pretend play: From theory to therapy. Child Language Teaching \& Therapy, 18(3), 233-255.

Leighton, C.J. (1992a). Juego de compartimiento dos tableros. En C.J. Leighton (Eds.), El desarrollo social en los niños pequeños: Egocentrismo y altruismo (pp. 117-121). Barcelona: Gedisa.

Leighton, C.J. (1992b). Juego de compartimiento con un compañero (pasas). En C.J. Leighton (Eds.), El desarrollo social en los niños pequeños: Egocentrismo y altruismo (pp. 121-123). Barcelona: Gedisa.

McCune, L., \& Zanes, M. (2001). Learning, attention, and play. En S. Golbeck (Ed.), Psychological perspectives on early childhood education (pp. 92-106). Mahwah, NJ: Lawrence Erlbaum.

Mikami, A.Y., Boucher, M.A., \& Humphreys, K. (2005). Prevention of peer rejection through a classroom-level intervention in middle school. Journal of Primary Prevention, 26(1), 5-23.

Portellano, J.A., Mateos, R., Martínez Arias, R., Granados, Mª J., \& Tapia A. (2000). Cuestionario de madurez neuropsicológica infantil CUMANIN. Madrid: TEA. 
Riojas-Cortez, M. (2001). Preschooler's funds of knowledge displayed through sociodramatic play episodes in a bilingual classroom. Early Childhood Education Journal, 29(1), 35-40.

Rolfe, S.A. (1991). The effects of prior experience in early childhood programs and play setting on social behavior and play in preschoolers. Early Child Development \& Care, 72, 23-37.

Rosberg, M. (1994). Enhancing learning through sociodramatic play. (ERIC Document Reproduction Service No. ED 387271).

Sarle, P.M. (2000). El juego dramático, la educación infantil y el aprendizaje escolar. Psykhe: Revista de la Escuela de Psicología, 9(2), 41-53.

Secadas, F. (1992). Escala Observacional del Desarrollo EOD. Madrid: TEA. (trabajo original publicado en 1988).

Seider, C.K. (2002). Factors affecting the frequencies of social behaviors and social goals of preschoolers across three types of dramatic play in an urban setting. Dissertation Abstracts International: Section A: Humanities \& Social Sciences, 62(11-A), 3685.

Shores, R.E., Huster, P., \& Strain, P.S. (1976). Effects of teacher presence and structured play on childchild interaction among handicapped preschool children. Psychology in the Schools, 13, 171-175.

Zan, B., \& Hildebrandt, C. (2003). First graders' interpersonal understanding during cooperative and competitive games. Early Education and Development, 14(4), 397-410. 